\section{College Members whose deaths were reported at Council meetings between October 2016 and October 2018}

Amara, Ibrahim Benjamin, Member, Kingston, Ontario, Canada Ainslie, John Archibald, Member, Canterbury, UK

Aungle, Peter George, Fellow, Dundee, UK

Bartrop, Roger Whitworth, Member, Roseville, New South

Wales, Australia

Bearcroft, John Stephen, Fellow, Lymington, Hampshire, UK

Bech, Per, Honorary Fellow, Rungsted, Denmark

Behrendt, Ralf-Peter, Member, Le Vauquiedor, St. Martin, Guernsey

Berry, Colin, Fellow, Finham, Coventry, UK

Berstock, Reva Leah, Member, Hampstead, London, UK

Bindal, Kanwal Krishan, Member, Omagh, UK

Brodie, Harlow Keith Hammond, Fellow, Durham, North

Carolina, USA

Black, Angus William, Fellow, Leven, Fife, UK

Bomford, John Powell, Member, Bendigo, Victoria, Australia

Brash, David Laurence, Member, Speers Point, New South

Wales, Australia

Brown, Alexander Campbell, Fellow, Clifton, Bristol, UK

Bruggen, Peter, Fellow, UK

Buchan, Terry, Fellow, Lesmurdie, Western Australia, Australia

Burke, Terence, Member, Fintry, Turriff, UK

Calwell, William Perry Kirkwood, Fellow, London, UK

Campbell, Maria Theresa Goretti T., Fellow, Penrith, Cumbria,

UK

Carlish, Sidney, Member, Moseley, Birmingham, UK

Carson, James Alexander, Fellow, Melbourne, Victoria,

Australia

Colville, John Robertson, Member, Dorchester, Dorset, UK

Davies, Roy James, Fellow, Burry Port, Wales, UK

De Alwis, Kithsiri Hemapala, Member, Kidlington, UK

Denford, John Douglas, Fellow, London, UK

Dolan, Robert, Member, UK

Duncan, Laura, Member, Inverurie, Aberdeenshire, UK

El-Bakly, Mohammad Madian, Member, London, UK

Farmer, Christopher John, Fellow, Vale, Guernsey

Enwere, Sampson Chukwuemeka, Affiliate, Milton Keynes, UK

Evans, Myrddin, Fellow, Llanddew, Brecon, UK

Famuyiwa, Oluwole Olumakinde, Fellow, Yaba, Lagos State

Nigeria

Fine, Eric William, Member, Elkins Park, Pennsylvania, USA

Fink, Klaus Philip, Fellow, Hamburg, Germany

Flanagan, William Laurence, Fellow, Tullibardine, Auchterarder, UK

Flynn, Thomas Gerard, Member, Omagh, UK

Fottrell, Eamonn, Fellow, London, UK

Gallagher, Melanie Ellen, Member, Rathgar, Dublin, Ireland

Galvin, Fionnuala Delia Maria, Member, Moycullen, Co Galway, Ireland

Ghoshal, Jogeschandra, Member, Kirkintilloch, Glasgow, UK

Gledhill, Maureen Thelma, Member, London, UK

Green, Elizabeth Aimee, Member, UK

Hardman, Anthony Eric, Fellow, Southport, Merseyside, UK

Heaton, John Moorhouse, Affiliate, London, UK

Hertzog, Jeanne Lovell, Foundation Member, Emsworth, UK
Higginson, John Christopher, Member, Banstead, UK

Hoare, Peter, Fellow, Henley-On-Thames, Oxfordshire, UK Hodgson, Oliver Ernest Fenner, Fellow, Histon, Cambridge, UK Irvine, George Murray, Member, Sudbury, Ontario, Canada James, Basil, Fellow, Townsville, Queensland, Australia Jayawant, Girija Sandeep, Affiliate, Marston, Oxford, UK Jimenez Leiva, David, Member, Pozuelo De Alacon, Madrid, Spain

Jones, Dilys Mair, Fellow, Old Windsor, Windsor, UK

Jones, Kingsley, Fellow, Loddon, Norwich, UK

Kane, Eileen Patricia, Member, Londonderry, UK

Kaplan, Theodore Harry, Member, Mount Carmel, Haifa, Israel Kesharwani, Shailesh Kumar, Affiliate, Bolton, Lancashire, UK Klasen, Henrika, Member, Haarlem, The Netherlands Kornan, Paul Jacob, Member, Melbourne, Victoria, Australia Lawler, Matthew Patrick Gerald, Member, Letterkenny, Co Donegal, Ireland

Lee, Catherine Ishbel Mackenzie, Member, Wexford, Ireland Lekh, Sudesh Kumar, Member, Banstead, Surrey, UK Leuvennink, Johannes Cornelius, Fellow, Dumfries, UK Lewis, Dawn Christine, Chair - Carer's Forum, Hereford, Herefordshire, UK

Liebling, Leonard Irving, Fellow, Sutton Coldfield, UK Lucas, Christopher James, Fellow, Letchworth Garden City, Hertfordshire, UK

Lutchmun, Nemnarainsingh, Member, London, UK

MacBride, Timothy P, Affiliate, Letterkenny, Co Donegal, Ireland

MacCallum, William Andrew Gordon, Fellow, Belfast, UK

McConnell, William Brian, Fellow, Newtown Abbey, UK

Mahmoud, Ahmed Mohamed Ahmed, Specialist Associate,

Nantwich, Cheshire, UK

Mahendran, Chelladurai, Affiliate, Harrow, UK

Malitz, Sidney, Fellow, Scarsdale, New York, USA

Manukulasuriya, Susil, Member, Aylesbury, UK

Maule, Rachel Emily, Member, Auckland, New Zealand

Mitchell, Alexander Ross Kerr, Fellow, Cambridge, UK

Nanayakkara, Vitharana Aratchi Gamini, Member, Oxford, UK

$\mathrm{Ng}$, Hon-Shing, Member, Tuen Mun, Hong Kong

Oakeshott, Simon, Member, Great Shelford, Cambridge, UK

Ogunde, Cheyvonne Charlene, Member, Wibsey, Bradford, UK

Olamosu, Abayomi, Affiliate, Yeovil, Somerset, UK

Padamsee, Kabir, Fellow, Mill Hill, Brentwood, UK

Parker, Robert Renshaw, Member, Knowsley Village, UK

Pierce, Chester Middlebrook, Honorary Fellow, Jamaica Plain,

Massachusetts, USA

Pilkington, Pamela, Member, London, UK

Pokorny, Michael Robert, Fellow, Mapperley Park, Nottingham, UK

Prentice, Rachel, Pre-Membership Psychiatric Trainee, Fulwood, Preston, UK

Priest, Robert George, Fellow, Iver, UK

Pugh, Caroline, Member, Holywood, Co Down, UK

Quinn, John Francis, Member, Monkstown, Co Dublin, Ireland

Raju, Sutharsan, Member, Bedford, UK

Reddie, Ethel Mary, Member, Bromsgrove, UK

Reveley, Adrianne Moore, Fellow, London, UK

Ritson, Roger, Affiliate, Douglas, Isle of Man

Roper, Peter Digby Lewington, Member, Montreal, Quebec,

Canada 
Scholefield, Charles, Member, Uldale, Wigton, UK Shepperd, Margaret Joyce, Affiliate, Port Navas, Falmouth, UK Stamp, Edwin Frederick Charles, Member, Mount Evelyn,

Victoria, Australia

Stanley, Ann Katharine, Fellow, Thorpe, Norwich, UK

Strickland, Paul Laurence, Fellow, Manchester, UK

Timol, Sulaiman Ahmed, Affiliate, Thorpe Bay, Southend on Sea, UK

Toms, David Anthony, Fellow, Tipton St. John, Sidmouth, UK Walczak, Paul Martin, Affiliate, Rochdale, Lancashire, UK

Walker, Derek Lindsay, Fellow, Painswick, Stroud, UK

Watt, Kerry Fiona, Member, Richmond, North Yorkshire, UK

Watts, Terence Christopher, Member, Glanamman,

Ammanford, UK

Westerholm, Ronald, Fellow, Bishopston, Swansea, UK

Whewell, Peter John, Fellow, Gosforth, Newcastle upon Tyne, UK

Williams, Akintunde Olufolahan, Member, Liverpool, UK
Wilson, Lesley, Member, St. Lawrence, Jersey

Winn, Pauline Imogen Margaret, Member, Brentford, UK Wool, Rosemary Jane, Fellow, Aston Clinton, Aylesbury, UK Woolridge, James Bancroft, Member, Malanda, Queensland, Australia

Worters, Alastair Robin, Fellow, Eyemouth, UK

doi:10.1192/bjb.2018.95

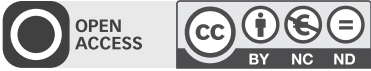

(c) The Royal College of Psychiatrists 2019. This is an Open Access article, distributed under the terms of the Creative Commons Attribution-NonCommercialNoDerivatives licence (http://creativecommons.org/licenses/by-nc-nd/4.0/) which permits non-commercial re-use, distribution, and reproduction in any medium, provided the original work is unaltered and is properly cited. The written permission of Cambridge University Press must be obtained for commercial reuse or in order to create a derivative work. 